\title{
Intravenous fentanyl during shoulder arthroscopic surgery in the sitting position after interscalene block increases the incidence of episodes of bradycardia hypotension
}

\author{
Seok Young Song ${ }^{1}$, Sang Hyuk Son ${ }^{1}$, Si Oh Kim², and Woon Seok Roh ${ }^{1}$ \\ Department of Anesthesiology and Pain Medicine, ${ }^{1}$ School of Medicine, Catholic University of Daegu, ${ }^{2}$ School of Medicine, \\ Kyungpook National University, Daegu, Korea
}

Background: Episodes of bradycardia hypotension (BH) or vasovagal syncope have a reported incidence of $13-29 \%$ during arthroscopic shoulder surgery in the sitting position after an interscalene block (ISB). This study was designed to investigate whether intravenous fentanyl during shoulder arthroscopy in the sitting position after ISB would increase or worsen the incidence of BH episodes.

Methods: In this prospective study, 20 minutes after being in a sitting position, 160 patients who underwent ISB were randomized to receive saline ( $\mathrm{S}, \mathrm{n}=40), 50 \mu \mathrm{g}$ of fentanyl (F-50, $\mathrm{n}=40$ ), $100 \mu \mathrm{g}$ of fentanyl (F-100, $\mathrm{n}=40$ ) or $30 \mathrm{mg}$ of ketorolac $(\mathrm{K}-30, \mathrm{n}=40)$ randomly. We assessed the incidence of $\mathrm{BH}$ episodes during the operation and the degree of maximal reduction (Rmax) of blood pressure (BP) and heart rate (HR).

Results: The incidence of BH episodes was $10 \%, 15 \%, 27.5 \%$ and $5 \%$ in the S, F-50, F-100 and K-30 groups, respectively. Mean Rmax of systolic BP in the F-100 group was significantly decreased as compared to the S group $(-20.0 \pm 4.5$ versus $-6.3 \pm 1.6 \%, P=0.004)$. Similarly, mean Rmax of diastolic BP in the F-100 group was also significantly decreased $(\mathrm{P}=0.008)$ as compared to the $\mathrm{S}$ group.

Conclusions: These results suggest that fentanyl can increase the incidence of $\mathrm{BH}$ episodes during shoulder arthroscopic surgery in the sitting position after ISB. (Korean J Anesthesiol 2011; 60: 344-350)

Key Words: Bradycardia, Fentanyl, Hypotension, Interscalene block, Vasovagal syncope.

Received: May 25, 2010. Revised: 1st, June 14, 2010; 2nd, September 28, 2010; 3rd, October 15, 2010; 4th, October 27, 2010. Accepted: October 27, 2010. Corresponding author: Woon Seok Roh, M.D., Ph.D., Department of Anesthesiology and Pain Medicine, School of Medicine, Catholic University of Daegu, 3056-6, Daemyung 4-dong, Nam-gu, Daegu 705-718, Korea. Tel: 82-53-650-4522, Fax: 82-53-650-4517, E-mail: usno@cu.ac.kr (c) This is an open-access article distributed under the terms of the Creative Commons Attribution Non-Commercial License (http:// creativecommons.org/licenses/by-nc/3.0/), which permits unrestricted non-commercial use, distribution, and reproduction in any medium, provided the original work is properly cited. 


\section{Introduction}

Interscalene brachial plexus block (ISB) is a regional anesthetic technique that provides anesthesia and analgesia to the shoulder. ISB has been shown to be successful when performed by dedicated anesthesiologists, but resistance to its use still exists because of cardiovascular instability such as episodes of bradycardia and/or hypotension (BH) as well as vasovagal syncope which is seen in $13-29 \%$ of patients undergoing shoulder arthroscopy in the sitting position after ISB [1-4]. The proposed mechanism is the Bezold-Jarisch reflex (BJR), triggered by reduced cardiac venous return in combination with affective mechanisms such as pain or fear [5]. Venous pooling and high sympathetic tone (induced by $\beta$-adrenergic effects of endogenous and exogenous catecholamines) occurs during shoulder surgery in the seated position, which leads to an underfilled, hypercontractile left ventricle and consequential activation of the parasympathetic nervous system. The end result is a transient, reversible, profound arterial vasodilation and bradycardia [2-6]. Based on these assumptions, several contributing factors for the occurrence of $\mathrm{BH}$ episodes have been suggested, such as perioperative use of epinephrine in local anesthetics for ISB, the use of $\beta$-blockers and preoperative hydration $[3,4]$. Unfortunately, there is no definite evidence that the cardio-inhibitory receptors or the BJR per se have a primary role in this type of syncope [7]. Moreover, in the retrospective study [8], intravenous fentanyl supplementation due to incomplete block was a new and possible contributing factor for the occurrence of $\mathrm{BH}$ episodes during shoulder arthroscopic surgery in the sitting position after ISB.

Fentanyl is a commonly used synthetic opiate and can evoke cardiovascular instability by neurologic and cardiac mechanisms [9-11]. In addition, there is some evidence that endogenous opioids, which are important neurotransmitters in nucleus tractus solitaries, are involved in syncopal reaction in animals [12]. Therefore, in the current study, we tested the hypothesis that intravenous fentanyl would increase the incidence of $\mathrm{BH}$ episodes during shoulder arthroscopy in the sitting position after ISB.

\section{Materials and Methods}

After obtaining approval from our institutional review board and written informed consent from 160 American Society of Anesthesiologists Physical Status (ASA PS) I or II patients who were to undergo shoulder arthroscopy in the sitting position after ISB, patients were assigned to one of four groups receiving either saline, $50 \mu \mathrm{g}$ of fentanyl, $100 \mu \mathrm{g}$ of fentanyl or $30 \mathrm{mg}$ of ketorolac by using simple random sampling. All patients received intramuscular premedication with $0.05 \mathrm{mg} / \mathrm{kg}$ of midazolam 30 minutes before going to the operating room. ISB using Winnie's technique was performed in the preoperative area or the operating room by an attending anesthesiologist. Patients were in the supine position with the head turned to the opposite side to be blocked. The interscalene groove was identified at the level of the cricoid cartilage, and the patient was prepped in an aseptic manner with iodine topical solution. Using a 25-gauge needle, 0.5 to $1 \mathrm{ml}$ of $1 \%$ lidocaine was injected subcutaneously to form a skin wheal. A sterile 24-gauge, 50mm insulated block needle (Braun Contiplex Block set; Braun, Melsungen, Germany) was used with the multiplex nerve stimulator (B. Braun, Melsungen, Germany). The initial current output of the nerve stimulator was set at $1.0 \mathrm{~mA}$. The insulated needle was advanced through the interscalene groove until a contraction of the biceps or deltoid muscle (or both), whichever appeared first, was elicited. The needle position was then adjusted until a twitch of either biceps or deltoid muscle group could still be elicited with a current output $<0.5 \mathrm{~mA}$, after which $20 \mathrm{ml}$ of $0.75 \%$ ropivacaine and $20 \mathrm{ml}$ of $1 \%$ lidocaine or mepivacaine was injected. After administering the local anesthetics, the degree of sensory blockade was evaluated by a pin prick test 15 minutes after injection. After the ISB was performed, patients received oxygen via a nasal cannulae at a rate of $2-4 \mathrm{~L} / \mathrm{min}$ and were monitored with continuous pulse oximetry, a noninvasive blood pressure (BP) device that was cycled every 5 minutes, with more frequent measurements as indicated, and continuous electrocardiography. They were then placed in the sitting or "beach chair" position, which could be achieved by elevating the back of the operating room table to $60-80^{\circ}$ and flexing the knees and hips to $90^{\circ}$ with the patients' feet resting firmly on a footboard. The study drug or saline was administered by bolus injection 20 minutes after positioning by the anesthesiologist who performed the ISB; another anesthesiologist analyzed the HR and BP trends. Patients with a history of coronary artery disease, cardiac conduction defects, diabetes, or asthma were excluded from the study. Patients receiving $\beta$-blockers or calcium channel blockers were also excluded. All patients underwent arthroscopic shoulder surgery for a variety of preoperative diagnoses, including supraspinatus tendon tear, rotator cuff tear, shoulder instability, labral tear, etc. The arthroscopic irrigating solution did not contain epinephrine $(1: 2,000,000)$ in an isotonic sodium chloride solution. Sedation consisted of midazolam and/or fentanyl at the discretion of the anesthesiologist. A BH episode was defined as any decrease in heart rate $(\mathrm{HR})<50 \mathrm{bpm}$, and/or any decrease of systolic BP (SBP) $<100 \mathrm{mmHg}$, with analysis of the HR and BP trends. This event also was accompanied by intraoperative treatment with atropine or ephedrine by the attending anesthesiologist. The cardiovascular parameters of each five minute-interval were then expressed as a percent change from the initial values in 
the sitting position or after intravenous injection of study drug. To investigate the effect of each drug on the cardiovascular variables, we also analyzed the maximal response (Rmax) of the percent change in either HR or BP from the initial values in the sitting position. Rmax of HR, SBP or diastolic BP (DBP) was defined as the Rmax of declines in the percent change of HR, SBP or DBP as compared with the initial values taken in the sitting position. It was also obtained within the limit of safety for ethical reasons. The Rmax time of HR, SBP or DBP which was defined as the time to reach Rmax for HR, SBP or DBP in the sitting position was also obtained. Statistical analysis consisted of ANOVA for a comparison of age, height, weight, time intervals, amount of fluid and Rmax or Rmax time for HR or BP. Chi-square analysis was performed to compare ASA PS, gender, type and site of surgery, and intraoperative medications. The percent changes of HR and SBP from injection of study drugs were compared with repeated-measure ANOVA. Statistical analyses were performed using SPSS 14.0.2 (Inc., Chicago, Illinois, USA). A P value of $<0.05$ was considered statistically significant. All data were reported as mean \pm SD.

\section{Results}

One hundred sixty patients were randomized to the three groups. No patients experienced any symptoms suggestive of complications from ISB such as spinal, epidural or intravascular injection of local anesthetics. Demographic data are presented in Table 1 . There were no differences among groups with regard to ASA PS, age, gender, height, weight, preoperative diagnosis and operation site (Table 1). Anesthetic characteristics and anesthetic related complications are shown in Table 2 . The ratio of ISB site, NPO time, readiness to time of surgery (defined as the time from the end of block to the operation start), the duration of anesthesia and surgery, and the amount of intraoperative fluids were not statistically different among the four groups. Eleven patients (27.5\%) in the F-100 group received ephedrine to treat hypotension which was significantly higher than the S group (10\%). Five patients in the F-50 group and 3 patients in F-100 group received atropine to treat bradycardia. However, there was no difference in a comparison for the use of midazolam or ondansetron. The maximal response (Rmax) to a decline in HR or BP is shown in Table 3. The incidence of BH episodes was $10 \%, 15 \%, 27.5 \%$ and $5 \%$ in the S, F-50, F-100 and K-30 groups, respectively. Initial cardiovascular values in the sitting position had no significant differences among the four groups. Mean Rmax of SBP in F-100 group was significantly decreased when compared to the $S$ group $(-6.3 \pm 1.6 \%$ versus $-20.0 \pm 4.5$ for S and F-100, respectively; $\mathrm{P}<0.05)$. Similarly, mean Rmax for DBP in the F-100 group was also significantly decreased $(\mathrm{P}<0.05)$ when compared to the $\mathrm{S}$ group. However, mean Rmax of HR in F-100 group was not significantly decreased when compared to the $S$ group $(-6.6 \pm 1.7 \%$ versus $-9.8 \pm 2.1$ for S and F-100, respectively; P > 0.05). The Mean Rmax for HR or BP in the K-30 group had a similar pattern than the $\mathrm{S}$ group. In addition, all groups were comparable with regard to their Rmax time for HR, SBP, or DBP (Table 3). Percent

Table 1. Demographic Data

\begin{tabular}{|c|c|c|c|c|}
\hline & $\begin{array}{l}\text { Saline } \\
(\mathrm{n}=40)\end{array}$ & $\begin{array}{c}\text { F-50 } \\
(\mathrm{n}=40)\end{array}$ & $\begin{array}{c}\text { F-100 } \\
(n=40)\end{array}$ & $\begin{array}{c}\mathrm{K}-30 \\
(\mathrm{n}=40)\end{array}$ \\
\hline ASA PS (I/II) & $34 / 6$ & $36 / 4$ & $37 / 3$ & $33 / 7$ \\
\hline Age (yr)* & $48.1 \pm 2.5$ & $47.4 \pm 2.7$ & $47.5 \pm 2.6$ & $51.8 \pm 2.3$ \\
\hline $\operatorname{Sex}(F / M)$ & $24 / 16$ & $30 / 10$ & $22 / 18$ & $21 / 19$ \\
\hline Height $(\mathrm{cm})^{*}$ & $164.9 \pm 1.3$ & $163.6 \pm 1.5$ & $167.3 \pm 1.6$ & $165.6 \pm 1.4$ \\
\hline Weight $(\mathrm{kg})^{*}$ & $62.4 \pm 1.9$ & $63.0 \pm 1.5$ & $67.6 \pm 2.0$ & $65.3 \pm 2.0$ \\
\hline \multicolumn{5}{|l|}{ Preoperative diagnosis } \\
\hline SST tear & 6 & 16 & 11 & 22 \\
\hline Shoulder SLAP & 1 & 4 & 2 & 2 \\
\hline Shoulder instability & 5 & 5 & 6 & 1 \\
\hline Recurrent dislocation & 0 & 2 & 0 & 2 \\
\hline Rotator cuff tear & 26 & 6 & 16 & 9 \\
\hline Pyogenic arthritis & 1 & 2 & 0 & 1 \\
\hline Calcified tendinitis & 0 & 2 & 0 & 1 \\
\hline Anterior labral tear & 0 & 3 & 3 & 2 \\
\hline AC joint arthritis & 0 & 0 & 2 & 0 \\
\hline Subacapular tear & 2 & 0 & 0 & 0 \\
\hline \multicolumn{5}{|l|}{ Operation site } \\
\hline Right/left & $27 / 13$ & $28 / 12$ & $29 / 11$ & $20 / 10$ \\
\hline
\end{tabular}

*Values are mean \pm SEM. F-50, F-100 and K-30 indicate the group of injection with $50 \mu \mathrm{g}$ or $100 \mu \mathrm{g}$ of fentanyl or 30 mg of ketorolac. ASA PS indicates American Society of Anesthesiology physical status. 
Table 2. Anesthetic Characteristics and Anesthetic Related Complications

\begin{tabular}{|c|c|c|c|c|}
\hline & $\begin{array}{l}\text { Saline } \\
(\mathrm{n}=40)\end{array}$ & $\begin{array}{c}\text { F-50 } \\
(n=40)\end{array}$ & $\begin{array}{c}\text { F-100 } \\
(\mathrm{n}=40)\end{array}$ & $\begin{array}{c}\mathrm{K}-30 \\
(\mathrm{n}=40)\end{array}$ \\
\hline Site of ISB (right/left) & $24 / 16$ & $30 / 10$ & $22 / 18$ & $21 / 19$ \\
\hline NPO time (min) & $726.6 \pm 44$ & $706.5 \pm 40$ & $699.4 \pm 32$ & $624.6 \pm 34$ \\
\hline Time from block to op. start (min) & $39.5 \pm 2.6$ & $41.1 \pm 5.3$ & $44.6 \pm 4.5$ & $55.1 \pm 3.8$ \\
\hline Duration of anesthesia (min) & $149.7 \pm 7.2$ & $140.1 \pm 10.7$ & $142.4 \pm 10.0$ & $163.7 \pm 9.7$ \\
\hline Duration of surgery (min) & $110.1 \pm 6.2$ & $99.0 \pm 8.5$ & $97.9 \pm 7.6$ & $108.7 \pm 8.7$ \\
\hline Amount of fluids (ml) & $446.7 \pm 46$ & $480.0 \pm 82$ & $419.0 \pm 48$ & $592.8 \pm 87$ \\
\hline \multicolumn{5}{|l|}{ Intraoperative medication } \\
\hline Ephedrine & 4 & 6 & $11^{*}$ & 2 \\
\hline $5 \mathrm{mg}$ & 0 & 1 & 1 & 0 \\
\hline $10 \mathrm{mg}$ & 3 & 5 & 9 & 1 \\
\hline$>15 \mathrm{mg}$ & 1 & 0 & 1 & 1 \\
\hline Atropine $(0.5 \mathrm{mg})$ & 0 & 5 & 3 & 0 \\
\hline Midazolam (1-2 mg) & 0 & 0 & 0 & 2 \\
\hline Ondansetron (4-8 mg) & 1 & 2 & 2 & 1 \\
\hline \multicolumn{5}{|l|}{ Intraoperative adverse events } \\
\hline Hypotension & 4 & 6 & $11^{*}$ & 2 \\
\hline Bradycardia & 0 & 5 & 3 & 0 \\
\hline Hypotension and bradycardia & 0 & 5 & 3 & 0 \\
\hline Nausea/vomiting & 1 & 2 & 2 & 1 \\
\hline
\end{tabular}

Values are mean \pm SEM. F-50, F-100 and K-30 indicate the group of injection with $50 \mu \mathrm{g}$ or $100 \mu \mathrm{g}$ of fentanyl or $30 \mathrm{mg}$ of ketorolac . $* \mathrm{P}<0.05$ versus saline group.

Table 3. Maximal Response (Rmax) to Decline of Heart Rate or Blood Pressure

\begin{tabular}{lcccc}
\hline & $\begin{array}{c}\text { Saline } \\
(\mathrm{n}=40)\end{array}$ & $\begin{array}{c}\text { F-50 } \\
(\mathrm{n}=40)\end{array}$ & $\begin{array}{c}\text { F-100 } \\
(\mathrm{n}=40)\end{array}$ & $\begin{array}{c}\mathrm{K}-30 \\
(\mathrm{n}=40)\end{array}$ \\
\hline BH episodes & 4 & 6 & $-9.8 \pm 2.1$ & 2 \\
Rmax of heart rate (\%) & $-6.6 \pm 1.7$ & $-5.9 \pm 2.1$ & $30.3 \pm 2.3$ & $-8.2 \pm 1.5$ \\
Rmax time of heart rate (min) & $34.0 \pm 2.8$ & $38.1 \pm 2.8$ & $-20.0 \pm 4.5^{*,+}$ & $37.1 \pm 2.7$ \\
Rmax of SBP (\%) & $-6.3 \pm 1.6$ & $-13.1 \pm 2.4$ & $40.3 \pm 2.6$ & $-7.7 \pm 1.9$ \\
Rmax time of SBP (min) & $33.9 \pm 2.4$ & $37.6 \pm 3.1$ & $-22.3 \pm 4.6^{*}$ & $39.9 \pm 3.0$ \\
Rmax of DBP (\%) & $-8.5 \pm 1.8$ & $-15.0 \pm 2.5$ & $40.3 \pm 2.6$ & $-10.7 \pm 2.1$ \\
Rmax time of DBP (min) & $33.9 \pm 2.4$ & $37.6 \pm 3.1$ & $39.9 \pm 3.0$ \\
\hline
\end{tabular}

Values are mean \pm SEM. F-50, F-100 and K-30 indicate the group of injection with $50 \mu \mathrm{g}$ or $100 \mu \mathrm{g}$ of fentanyl or $30 \mathrm{mg}$ of ketorolac. BH episodes refer to bradycardia-hypotension episodes. Maximal response (Rmax) of heart rate (HR), systolic or diastolic blood pressure (SBP or DBP) is defined as the Rmax of declines in the percent change of HR, SBP or DBP. ${ }^{*} \mathrm{P}<0.05$ versus saline group. ${ }^{\dagger} \mathrm{P}<0.05$ versus ketorolac group.

change of HR after bolus injection of each drug in the F-100 group was also decreased, but not significantly ( $\mathrm{P}>0.05$; Fig. 1). Percent change of SBP after bolus injection of each drug in the F-100 group was significantly decreased as compared with those in group S (Fig. 2).

\section{Discussion}

The main purpose of this prospective study was to test the hypothesis that intravenous fentanyl would increase the incidence of $\mathrm{BH}$ episodes in arthroscopic shoulder surgery in the sitting position after ISB. The results of our study demonstrated that fentanyl can increase the incidence of $\mathrm{BH}$ episodes in a dose-dependent manner. To the best of our knowledge, this study is the first to evaluate whether fentanyl would be a contributing factor for the occurrence of $\mathrm{BH}$ episodes in the arthroscopic shoulder surgery after ISB.

One proposed mechanism for the occurrence of $\mathrm{BH}$ episodes is based on activation of the BJR $[2,5,6]$. Activation of the BJR initiates from an empty hypercontractile ventricle which causes stimulation of intramyocardial mechanoreceptors (C fibers) and produces an abrupt withdrawal of sympathetic outflow in addition to an increase of vagal tone resulting in bradycardia and hypotension. D'Alessio et al. reported [2] that the mechanism of the BJR during shoulder arthroscopic surgery was thought to be venous blood pooling (induced by the sitting position) and a heightened cardiac contractile state (induced by the $\beta$-adrenergic effects of epinephrine or isoproterenol) which results in reflex arterial vasodilation (mediated by activation of the parasympathetic nervous system) and a 


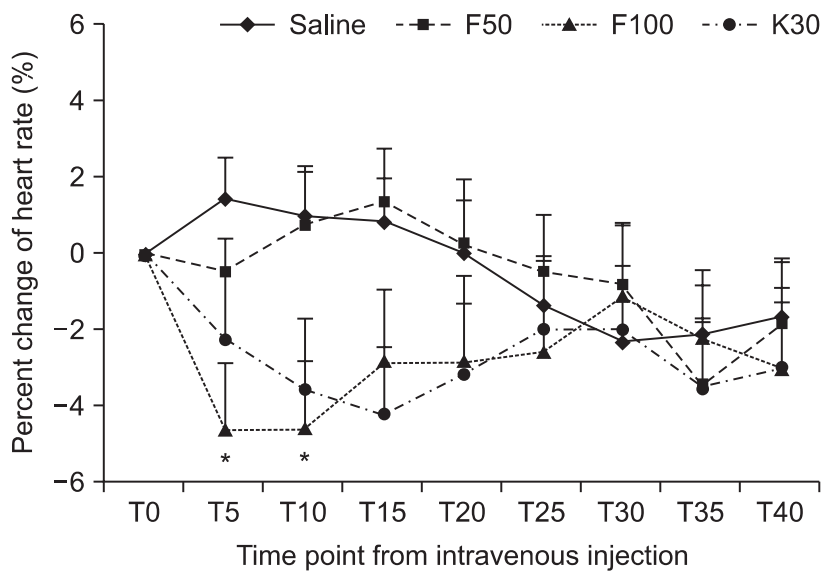

Fig. 1. Percent change of heart rate according to time point from the injection of study drug. Values are expressed as mean \pm SD. Tn: $n$ minutes after injection time. ${ }^{*} \mathrm{P}<0.05$ compared with saline group.

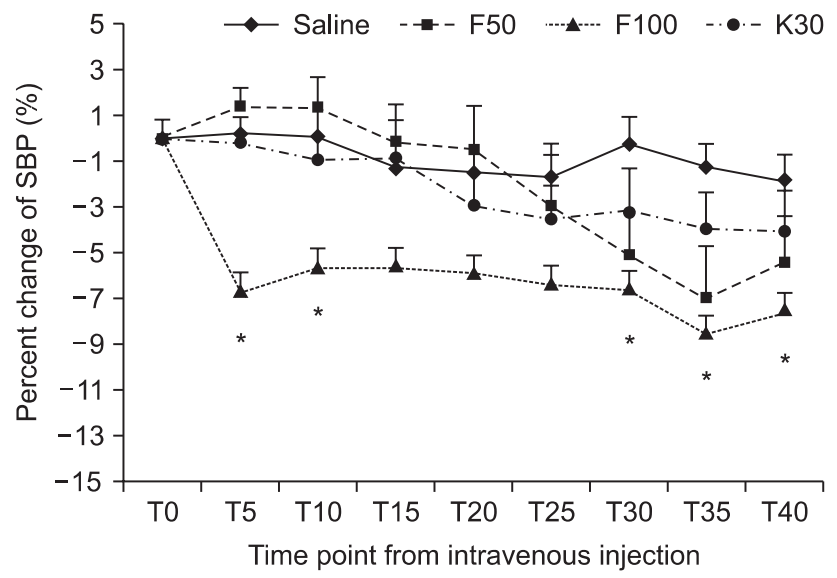

Fig. 2. Percent change of systolic blood pressure according to time point from the injection of study drug. Values are expressed as mean \pm SD. Tn: $\mathrm{n}$ minutes after injection time. ${ }^{*} \mathrm{P}<0.05$ compared with saline group.

would increase the incidence of $\mathrm{BH}$ episodes in the arthroscopic shoulder surgery at the sitting position after ISB. The present study demonstrated that $\mathrm{BH}$ episodes were increased in the F-100 group (27.5\%), but not the S (10\%), F-50 (15\%) or K-30 groups (5\%). For supplementation during regional anesthesia, an intravenous bolus of fentanyl $(1-3 \mu \mathrm{g} / \mathrm{kg})$ is frequently chosen because fentanyl in these dose ranges can produce potent, short lasting analgesia [13]. Although there are a few reports on the cardiovascular effects of IV supplemental doses of fentanyl, Larson et al. [14] demonstrated that IV fentanyl ( $3 \mu \mathrm{g} / \mathrm{kg}$ ) during the stimulation in the desflurane anesthesia decreased heart rate and arterial blood pressure, but with less than a $10 \%$ chance. In the current study, a reasonable dose (100 $\mu \mathrm{g}$ ) of fentanyl supplementation produced a marked decrease of arterial blood pressure with the mean percent change of about $20 \%$. These findings suggest that fentanyl-induced hypotension may be potentiated under certain conditions in the sitting position like shoulder arthroscopic surgery.

The effect of being in a sitting or beach-chair position during shoulder arthroscopic surgery is similar to postural changes from supine to a head-up tilt. These postural changes induce a gravity-driven venous blood pooling in the lower extremities and abdominal viscera, which cause a decrease in ventricular preload. Immediately on standing, the arterial pressure in the head and upper part of the body tends to fall. However, the falling pressure at the baroreceptor induces an immediate reflex, resulting in an increase of sympathetic tone and a decrease in vagal tone as a compensatory response. This predominant sympathetic efferent signal causes an increase in systemic vascular resistance, increased HR, and a heightened state of inotropy [15-17]. Under this condition, an intravenous bolus of fentanyl may reduce the compensatory response the current study that intravenous administration of fentanyl 
because fentanyl can cause opioid-induced hypotension or increased parasympathetic activity. In addition, it should be noted that ISB can produce hypotension via its side-effects including a spinal or epidural blockade and/or a stellate ganglionic block. Sympathetic blockade due to ISB may attenuate the compensatory response derived from a postural change to the sitting position [18]. Taking these findings into consideration, we can speculate that shoulder arthroscopic surgery is prone to develop fentanyl-induced hypotension.

It is well known that opioid-induced hypotension is mediated by neurologic, cardiac or hormonal mechanisms. In the neurologic mechanism, key areas of the brainstem that integrate cardiovascular responses and maintain cardiovascular homeostasis are the nucleus solitarius, the dorsal vagal nucleus, the nucleus ambiguus, and the parabrachial nucleus. The direct administration of $\mu$-agonists into the central nervous system of animals most commonly, but not always, produces hypotension and bradycardia [19]. Microinjections of excitatory amino acids made into the ventrolateral midbrain periaqueductal gray of the rat have revealed that neurons in this region integrate a reaction characterized by quiescence, hyporeactivity, hypotension and bradycardia [9]. In addition, Griffioen et al. [20] demonstrated that fentanyl acts on $\mu$-opioid receptors on cardiac vagal neurons in the nucleus ambiguus and neurons preceding them to reduce GABAergic neurotransmission and increase parasympathetic activity. In the cardiac mechanism, fentanyl produces little or no change in myocardial contractility [21]. However, fentanyl slows atrioventricular node conduction, and prolongs the RR interval, the atrioventricular node refractory period and purkinje fiber action potential duration $[10,22]$. In addition, pharmacologic studies evaluating alfentanil, fentanyl, and sufentanil in the dog demonstrate direct peripheral vessel smooth muscle relaxation [11]. In the hormonal mechanism, fentanyl does not stimulate histamine release [23].

Ketorolac is the most potent analgesic in the non-steroidal anti-inflammatory drug (NSAID) group available for human use and has a more favorable profile with respect to cardiorespiratory effects, causing less reduction in $\mathrm{BP}, \mathrm{HR}$, and respiratory rate than do potent short-acting opioids during general anesthesia [24,25]. Camu et al. [26] reported that ketorolac at either dose (10 mg or $90 \mathrm{mg}$ ) was not associated with any significant change in cardiac and hemodynamic parameters such as mean arterial pressure, pulmonary artery pressure and cardiac output. Our results also demonstrated that ketorolac had little effect on heart rate and SBP or DBP. Unfortunately, we could not measure the analgesic effect of ketorolac because of the powerful analgesia induced by ISB. However, ketorolac is an effective drug for the treatment of moderate to severe pain following minor or intermediate types of surgery [27]. It is therefore not surprising that the intraoperative use of intravenous NSAIDs alone or combination with midazolam provides adequate analgesia during the minor procedures like shoulder arthroscopic surgery. We also think that the use of intravenous NSAIDs provides a preemptive analgesia [28] and it reduces the amount of opioids intraoperatively. Because our results show a hemodynamic instability caused by intravenous bolus of $100 \mu \mathrm{g}$ of fentanyl, we suggest that intravenous $50 \mu \mathrm{g}$ of fentanyl or $30 \mathrm{mg}$ of ketorolac is a safe and effective starting dose for supplementation during shoulder arthroscopic surgery.

In summary, although BH episodes are unpredictable events, our results demonstrate that intravenous fentanyl can increase the incidence of $\mathrm{BH}$ episodes in the arthroscopic shoulder surgery at the sitting position after ISB. BH episodes are usually of minor significance, but in extreme cases, it may lead to cardiac arrest. Therefore, we suggest careful observation for patients who have received a shoulder arthroscopic surgery in the sitting position, where there are incomplete blocks and analgesics needed for supplementations after ISB, especially in the case of intravenous bolus administration of fentanyl.

\section{References}

1. Kahn RL, Hargett MJ. Beta-adrenergic blockers and vasovagal episodes during shoulder surgery in the sitting position under interscalene block. Anesth Analg 1999; 88: 378-81.

2. D'Alessio JG, Weller RS, Rosenblum M. Activation of the bezoldjarisch reflex in the sitting position for shoulder arthroscopy using interscalene block. Anesth Analg 1995; 80: 1158-62.

3. Liguori GA, Kahn RL, Gordon J, Gordon MA, Urban MK. The use of metoprolol and glycopyrrolate to prevent hypotensive/bradycardic events during shoulder arthroscopy in the sitting position under interscalene block. Anesth Analg 1998; 87: 1320-5.

4. Sia S, Sarro F, Lepri A, Bartoli M. The effect of exogenous epinephrine on the incidence of hypotensive/bradycardic events during shoulder surgery in the sitting position during interscalene block. Anesth Analg 2003; 97: 583-8.

5. Kinsella SM, Tuckey JP. Perioperative bradycardia and asystole: relationship to vasovagal syncope and the bezold-jarisch reflex. Br J Anaesth 2001; 86: 859-68.

6. Almquist A, Goldenberg IF, Milstein S, Chen MY, Chen XC, Hansen R, et al. Provocation of bradycardia and hypotension by isoproterenol and upright posture in patients with unexplained syncope. N Engl J Med 1989; 320: 346-51.

7. Campagna JA, Carter C. Clinical relevance of the bezold-jarisch reflex. Anesthesiology 2003; 98: 1250-60.

8. Seo KC, Park JS, Roh WS. Factors contributing to episodes of bradycardia hypotension during shoulder arthroscopic surgery in the sitting position after interscalene block. Korean J Anesthesiol 2010; 58: 38-44.

9. Keay KA, Crowfoot LJ, Floyd NS, Henderson LA, Christie MJ, Bandler R. Cardiovascular effects of microinjections of opioid agonists into the 'depressor region' of the ventrolateral periaqueductal gray 
region. Brain Res 1997; 762: 61-71.

10. Blair JR, Pruett JK, Introna RP, Adams RJ, Balser JS. Cardiac electrophysiologic effects of fentanyl and sufentanil in canine cardiac purkinje fibers. Anesthesiology 1989; 71: 565-70.

11. White DA, Reitan JA, Kien ND, Thorup SJ. Decrease in vascular resistance in the isolated canine hindlimb after graded doses of alfentanil, fentanyl, and sufentanil. Anesth Analg 1990; 71: 29-34.

12. Wallbridge DR, MacIntyre HE, Gray CE, Oldroyd KG, Rae AP, Cobbe SM. Role of endogenous opioids and catecholamines in vasovagal syncope. Eur Heart J 1996; 17: 1729-36.

13. White PF. Textbook of intravenous anesthesia. 1st ed. Baltimore, Williams and Wilkins. 1997, p 232.

14. Larson MD, Berry PD, May J, Bjorksten A, Sessler DI. Autonomic effects of epidural and intravenous fentanyl. Br J Anaesth 2007; 98 263-9.

15. Rubin AM, Rials SJ, Marinchak RA, Kowey PR. The head-up tilt table test and cardiovascular neurogenic syncope. Am Heart J 1993; 125: 476-82.

16. Grubb BP, Kimmel S. Head-upright tilt table testing. A safe and easy way to assess neurocardiogenic syncope. Postgrad Med 1998; 103: $133-8,140$

17. Nwosu EA, Rahko PS, Hanson P, Grogan EW Jr. Hemodynamic and volumetric response of the normal left ventricle to upright tilt testing. Am Heart J 1994; 128: 106-13.

18. Nakagawa M, Takahashi N, Iwao T, Yonemochi H, Ooie T, Hara M, et al. Evaluation of autonomic influences on QT dispersion using the head-up tilt test in healthy subjects. Pacing Clin Electrophysiol 1999; 22: 1158-63.

19. Feldman PD, Parveen N, Sezen S. Cardiovascular effects of leuenkephalin in the nucleus tractus solitarius of the rat. Brain Res
1996; 709: 331-6.

20. Griffioen KJ, Venkatesan P, Huang ZG, Wang X, Bouairi E, Evans C, et al. Fentanyl inhibits GABAergic neurotransmission to cardiac vagal neurons in the nucleus ambiguus. Brain Res 2004; 1007: 10915.

21. Mathews HM, Furness G, Carson IW, Orr IA, Lyons SM, Clarke RS. Comparison of sufentanil-oxygen and fentanyl-oxygen anaesthesia for coronary artery bypass grafting. Br J Anaesth 1988; 60: 530-5.

22. Royster RL, Keeler DK, Haisty WK, Johnston WE, Prough DS. Cardiac electrophysiologic effects of fentanyl and combinations of fentanyl and neuromuscular relaxants in pentobarbital-anesthetized dogs. Anesth Analg 1988; 67: 15-20.

23. Flacke JW, Flacke WE, Bloor BC, Van Etten AP, Kripke BJ. Histamine release by four narcotics: a double-blind study in humans. Anesth Analg 1987; 66: 723-30.

24. Murray AW, Brockway MS, Kenny GN. Comparison of the cardiorespiratory effects of ketorolac and alfentanil during propofol anaesthesia. Br J Anaesth 1989; 63: 601-3.

25. Ramirez-Ruiz M, Smith I, White PF. Use of analgesics during propofol sedation: a comparison of ketorolac, dezocine, and fentanyl. J Clin Anesth 1995; 7: 481-5.

26. Camu F, Van Overberge L, Bullingham R, Lloyd J. Hemodynamic effects of two intravenous doses of ketorolac tromethamine compared with morphine. Pharmacotherapy 1990; 10: 122S-6S.

27. Feld JM, Laurito CE, Beckerman M, Vincent J, Hoffman WE. Nonopioid analgesia improves pain relief and decreases sedation after gastric bypass surgery. Can J Anaesth 2003; 50: 336-41.

28. Norman PH, Daley MD, Lindsey RW. Preemptive analgesic effects of ketorolac in ankle fracture surgery. Anesthesiology 2001; 94: 599603. 